\title{
USO DA SEQUÊNCIA FLAIR-EPI NA ANÁLISE DA ESCLEROSE MESIAL TEMPORAL
}

\author{
Marcos Alberto da Costa Machado Júnior', Fabíola Goyanna², \\ Adriana Silva Matos², Verônica Aline Oliveira Barbosa' ${ }^{1}$, Lauro Conceição Vieira ${ }^{3}$
}

\begin{abstract}
RESUMO - O objetivo deste estudo é analisar as alterações morfológicas e de intensidade de sinal das regiões hipocampais em pacientes, com epilepsia temporal fármaco-resistente. Para tal, estudamos 8 pacientes com esclerose mesial temporal, utilizando aparelhagem de RM de 1,5T, com sequências Spin Eco - SE, Fast Spin Eco - FSE, Fluid Atenuation Inversion Recovery, com Eco Planar Imaging - FLAIR-EPI. Observamos a superioridade da sequência FLAIR na detecção do aumento da intensidade de sinal da região hipocampal, particularmente com cortes coronais, em relação às sequências $\mathrm{SE}$ e FSE, com a vantagem de ser uma técnica de rápida execução. A sequência STIR evidenciou adelgaçamento da cortical do hipocampo, na metade dos casos que apresentavam alteração de sinal.
\end{abstract}

PALAVRAS-CHAVE: esclerose mesial temporal, ressonância magnética, FLAIR-EPI.

\section{EPI-FLAIR sequence in the evaluation of mesial temporal sclerosis}

\begin{abstract}
The purpose of this study is to evaluate morpholologycal and signal intensity changes in the hippocampus in patients with medically intractable temporal lobe epilepsy. We studied 8 patients with mesial temporal sclerosis using a 1.5 -T MR and the following sequences Spin Eco- SE, Fast Spin Echo- FSE, Fluid Atenuation Inversion Recovery Echo Planar Imaging - FLAIR-EPI. We noticed a sensitive increase signal intensity on FLAIR- EPI sequences, particularly, in coronal images, than on SE and FSE sequences. The STIR sequence showed a cortical hippocampus atrophy in half of the cases, in whom signal abnormalities were present.
\end{abstract}

KEY WORDS: mesial temporal sclerosis, magnetic resonance imaging, FLAIR-EPI.

A expressão esclerose mesial temporal (EMT) foi introduzida, por Falconer e col., em 1964', para descrever a lesão caracterizada por perda neuronal e gliose envolvendo, principalmente, o hipocampo e a amígdala, ou ambos, notadamente as camadas de células piramidais do corno de Ammon e a camada granulada do giro denteado. Estende-se, ocasionalmente, para outras estruturas mesias temporais ou até por todo o lobo temporal, levando à atrofia e gliose generalizada. EMT, também conhecida como esclerose hipocampal, é a causa mais comum de epilepsia com origem ou envolvimento primário do lobo temporal. De difícil tratamento, ela está presente em cerca de $65 \%$ das lobectomias temporais ${ }^{2}$.

os pacientes com epilepsia fármaco-refratária, causada por EMT, têm apenas um método eficaz de tratamento: ressecção cirúrgica do hipocampo. A ressecção cirúrgica do hipocampo e do lobo temporal anterior podem curar a epilepsia, em mais de $90 \%$ desses pacientes, tornando-se imperativos os métodos de imagem. Eles vêm alterando, dramaticamente, a habilidade em identificar EMT, antes da cirur$\mathrm{gia}^{3,4}$.

O objetivo deste estudo é analisar as alterações morfológicas e de intensidade de sinal na ressonância magnética (RM) das regiões hipocampais de pacientes com epilepsia temporal farmaco-resistente (ETFR).

\section{MÉTODO}

Foram estudados 26 pacientes com ETFR, selecionados, clinicamente e com EEG, pelo Departamento de Neurologia do HSR .

Usamos aparelhagem de RM de 1,5T (GE.- Signa Horison LX.) e o protocolo de exame abaixo:

- FSE COR T2 (TR 4.500, TE 95), Bw $15,3 \mathrm{~mm}$ de espessura, gap de 1,0 mm;

- DP COR (TR 200, TE 35), 3 mm de espessura e 1,0 de gap;

- SE;

- $\quad$ SAG. (TR 500, TE 14), 3 mm, 1,5 Gap;

Estudo realizado no Setor de Neurorradiologia do Serviço de Bioimagem do Hospital São Rafael (HSR) - Monte Tabor (MT), Salvador Bahia: ${ }^{1}$ Neurorradiologista do Serviço de Bioimagem do HSR-MT; Hospital Salvador - IGASE; Fundação de Neurologia e Neurocirurgia Instituto do Cérebro - Salvador - Bahia; ${ }^{2}$ Residente em Radiologia do HSR-MT; ${ }^{3}$ Técnico do Serviço de Bioimagem do HSR/MT.

Recebido 18 Julho 2000, recebido na forma final 20 Outubro 2000. Aceito 30 Outubro 2000. 
- $\quad$ AX (TR 4.000, TE 102), 3 mm. , 1,5 Gap;

- $\quad$ AX (TR 2.200, TE 34), 3 mm. , 1,5 Gap;

- $\quad$ FLAIR EPI AX (TR 10.000, TI 2.200, TE120), 8 shots, bw $635 \mathrm{~mm}$, sem gap, duração de $2.45 \mathrm{~min}$;

- COR, (TR 1000, TI 2200 TE 120 ), 3mm, sem Gap;

- STIR - ( Short - tau Inversion Recovery) COR (TR 4.000, TE 28, TI 150); AX (TR 4.050, TE 28, TI 150);

Os cortes coronais orientavam-se no maior eixo do giro para-hipocampal, cortando-o em ângulo reto.

\section{RESULTADOS}

Dos 26 pacientes com ETFR estudados, 14 apresentavam gliose extra-hipocampal inespecífica, 2 astrocitomas, 1 displasia do lobo temporal e 1 defeito de migração cortical (micropoligiria), que foram excluídos por apresentarem etiologia secundária. Apenas 8 apresentavam achados compatíveis com EMT, utilizando-se todas as sequências, e 6 foram identificados nas sequências FSE e SE (Figs 1 e 2).

Em 6 casos (dos 8 com hiperintensidade de sinal na sequência FLAIR), observou-se atrofia hipocampal associada. Nos dois casos em que as sequências SE e FSE não mostraram alterações da intensidade de sinal, assinalamos alterações atróficas dos hipocampos.

A sequência STIR coronal, fotografada em positivo, mostrava-se superior a todas as outras, na detecção de alterações atróficas, devido à sua maior sensibilidade na diferenciação da substância branca e cinzenta (Figs. 1D e 2C).

A sequência FLAIR-EPI identificou com segurança hiperintensidade de sinal, no hipocampo, em todos os 8 casos, enquanto que, apenas, 6 foram identificados na FSE e na SE ( Figs. 1A a D).

\section{DISCUSSÃO}

A fisiopatologia da EMT não está completamente entendida. Postula-se que seja causada por dano no desenvolvimento cerebral durante a infância, como crise febril complicada e encefalite. $\mathrm{O}$ giro denteado danificado reorganiza-se, levando a um sistema sináptico com hipersensibilidade aberrante,

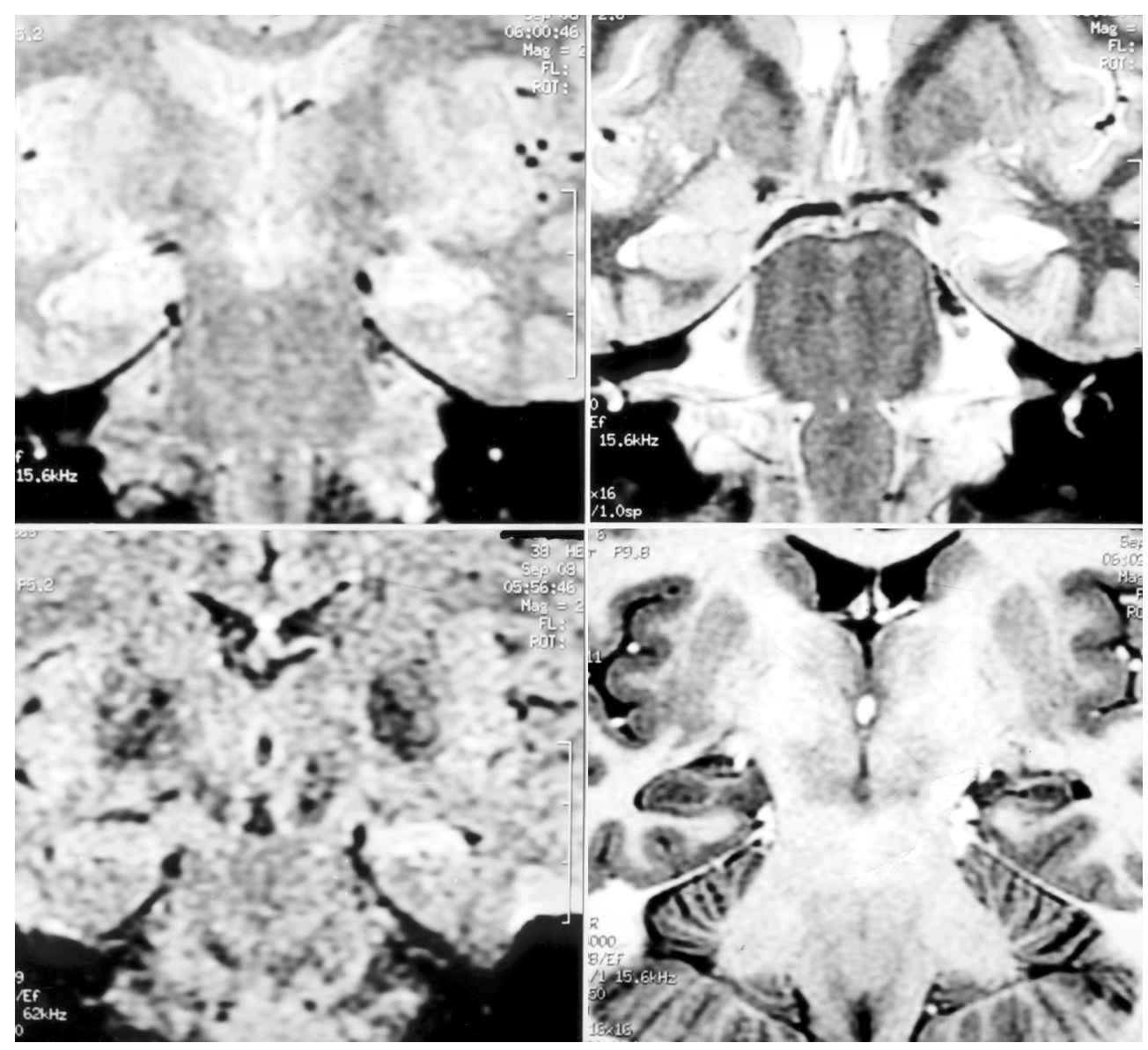

Fig 1. Esclerose mesial do hipocampo a esquerda. Cortes coronais com 3,0 mm de espessura. $\boldsymbol{A}$ - FSE DP, B - FSE T2, C - FLAIR-EPI e D - STIR fotografada em positivo. Evidencia a superioridade da sequência FLAIR-EPI (C), em relação a FSE DP (A) e FSE T2 (B). A atrofia hipocampal é evidente nas sequências STIR, fotografada em positivo (D). 


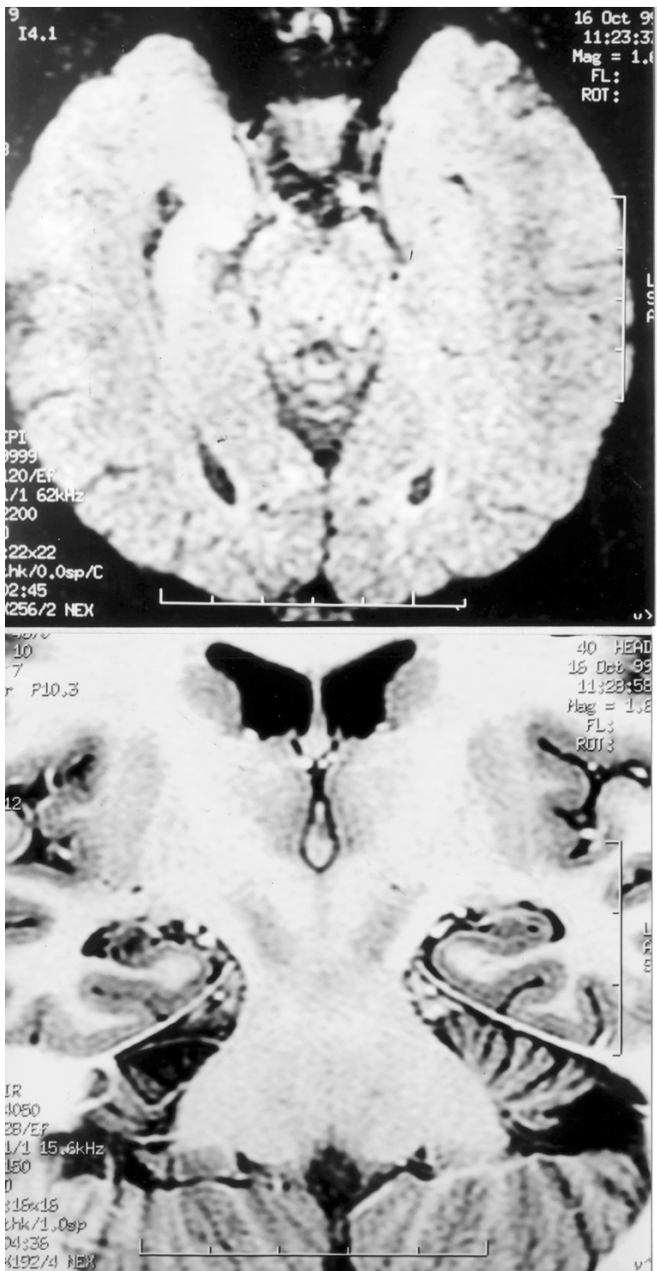

manifestando-se, clinicamente, com crises convulsivas recorrentes ${ }^{5}$. A esclerose hipocampal é lesão epileptogênica, confirmada por dados histopatológicos. Entretanto, sabe-se que, até $10 \%$ de um grupo controle, formado por indivíduos sem convulsão, apresentam tais alterações ${ }^{6}$.

Existe larga faixa de técnicas de imagem disponíveis para o diagnóstico e para localização da EMT. Esta inclui RM, usando inspeção visual simples, RM volumétrica do hipocampo, espectroscopia pela RM, tomografia computadorizada com emissão de fóton simples (SPECT), SPECT ictal, e tomografia com emissão de pósitrons (PET). Contudo, é a RM a técnica de imagem mais comumente usada. Os numerosos estudos de pacientes com epilepsia do lobo temporal têm demonstrado que a RM é um método seguro para localizar a origem da epilepsia do lobo temporal. A RM alcança até $93 \%$ de sensibilidade e $94 \%$ de especificidade, no estudo da EMT?.

O padrão de EMT, na RM, é uma atrofia do hipocampo, associada com hiperintensidade de sinal, nas sequências de longo TR limitada ao hipocampo3

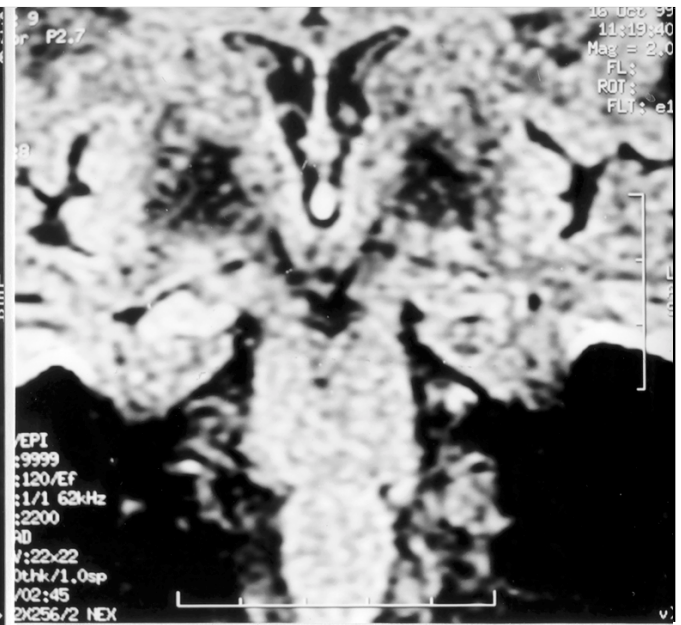

Fig 2. Esclerose mesial do hipocampo direito facilmente identificada na sequência FLAIR-EPI, pela sua marcada hiperintensidade de sinal (A e B). A atrofia hipocampal é vista em STR (C). É evidente o aumento do corno inferior adjacente. A - Axial FLAIR-EPI, B - Coronal FLAIR$E P I, C$ - Coronal STIR.

perda da definição das estruturas internas do hipocampo, hipointensidade em T1, esclerose de amígdalas e alterações do giro para-hipocampal. Entretanto, estima-se que em 70 a $90 \%$ dos pacientes com epilepsia focal crônica apresentem algum tipo de alteração estrutural do lobo temporal ${ }^{7}$.

A atrofia e a hiperintensidade são frequentemente consignadas como os dois achados primários da RM para EMT. Os portadores dessas alterações primárias da RM têm $70 \%$ a $90 \%$ de probabilidade de ficarem livres das crises com a lobectomia temporal. Por outro lado, na ausência desses sinais, os pacientes têm menos de $50 \%$ de probabilidade de beneficiarem-se com a cirurgia ${ }^{3,4}$.

Hiperintensidade de sinal, em T2, no hipocampo, também pode ser encontrada em tumor hipocampal, hamartoma, ou processo inflamatório, que produzam aparência similar àquela vista na EMT. Heinz e col. sugerem que, na presença de significativo aumento na intensidade de sinal, espera-se encontrar neoplasia, hamartoma ou cisto e, em pacientes com mínima hiperintensidade de sinal, $\mathrm{EMT}^{6}$. A hiperin- 
tensidade de sinal em T2 no hipocampo é a apresentação mais comum de EMT (sensibilidade 93\%), porém, a especificidade para confiança diagnóstica aumenta se associado com outras alterações, como atrofia do hipocampo ou do lobo temporal ipsilateral (de $74 \%$ para mais de $94 \%)^{8}$.

A atrofia hipocampal é outro importante achado na EMT, porém bastante dependente da experiência do observador. Está descrito que a atrofia tem sido diagnosticada em cerca de $80 \%$ a $85 \%$ dos casos, apenas pelo critério visual. A avaliação morfológica não é fácil, nos casos de alteração bilateral, tornandose menos complicada quando unilateral. Neste caso, o lado normal serve de parâmetro comparativo ${ }^{8}$.

Os achados secundários de RM ajudam no diagnóstico e na localização da EMT. E , em casos de anormalidades bilaterais do hipocampo, as alterações secundárias podem determinar o lado prioritário para ablação ${ }^{10}$.

A utilização do gadolínio paramagnético não está indicada no estudo da EMT, uma vez que esta entidade não se acompanha de quebra da barreira hemato-encefálica. O contraste paramagnético tem, entretanto, papel indispensável naqueles casos de convulsões secundárias a lesão de natureza tumoral, inflamatória ou vascular.

Introduziu-se, mais recentemente, a sequência FLAIR, capaz de fornecer imagens fortemente pesadas em $T 2$ e de eliminar o sinal do líquor, facilitando a detecção de anormalidades corticais ou periventriculares, possibilitando, assim, uma documentação mais sensível de alteração da intensidade de sinal do hipocampo, anulando os artefatos de efeito de volume parcial do líquor do corno inferior ${ }^{11-13}$. Fillipi e col. ${ }^{14}$ comparam as sequências Fast- FLAIR, FLAIRHASTE e FLAIR-EPI, na detecção de placas de esclerose múltipla, em 46 pacientes, evidenciando melhor qualidade de imagem nas sequências FLAIR-EPI e FLAIR-HASTE e ressaltam a importância da primeira, principalmente em pacientes não colaborativos, devido à sua rapidez. Nossos resultados confirmam estes dados, considerando que a FLAIR foi essencial em dois casos, nos quais as sequências FSE e SE nada revelaram.
O uso da sequência STIR (short-tau inversion recovery), fotografada em positivo, foi a mais útil na detecção de alterações atróficas dos hipocampos.

\section{Conclusão}

Os mais recentes estudos vêm demonstrando associação entre as alterações de intensidade de sinal e de volume do hipocampo. Em nosso estudo, a sequência FLAIR-EPI, com cortes coronais, mostrouse superior à FSE e à $\mathrm{SE}$, na detecção de alterações da intensidade de sinal do hipocampo, com duração de apenas 2,45 minutos. Em $80 \%$ dos casos, em que era visível alteração da intensidade de sinal, encontramos adelgaçamento da cortical do hipocampo, achado melhor identificado na sequência STIR, em todos os casos.

Enfim, alteração de intensidade de sinal e de volume sugerem patologia do hipocampo e são importantes na correta seleção de pacientes que podem ser submetidos à terapia cirúrgica.

\section{REFERÊNCIAS}

1. Falconer MA, Searafetinides EA, Corsellis JAN. Etiology and pathogenesis of temporal lobe epilepsy. Arch Neurol 1964;10:233-248.

2. Babb Tl, Brown WJ. Pathological finding in epilepsy. In Engel JJr (ed.) Surgical treatment of the epilepsies. New York: Raven Press; 1987:511-540.

3. Jack CJ Jr, Sharbrough FW, Cascino GD, Hirschorn KA, O'Brien PC, Marsh WR. Magnetic resonance image-based hippocampal volumetry: correlation with outcome after temporal lobectomy. Ann Neurol 1992;31:138-146.

4. Bronen R. MR of mesial temporal sclerosis: how much is enough? AJNR 1998;19:15-17.

5. DeLanerolle NC, Brines ML, Kim JH, Williamson A, Philips MF, Spencer DD. Neurochemical remodelling of the hippocampus in human temporal lobe epilepsy. Epilepsy Res 1992;9(Suppl):205-219.

6. Heinz ER, Crain BJ, Radtke RA, et al.. MR imaging in patients with temporal lobe seizures: correlation of results with pathologic findings. AJR 1990;155:581-586

7. Achten E, Boon P, De Poorter J, Calliauw L. An MR protocol for presurgical evaluation of patients with complex partial seizures of temporal lobe origin. AJNR 1995;16:1201-1213.

8. Lee DH, Gao FQ, Rogers JM, et al.. MR in temporal lobe epilepsy: analysis with pathologic confirmation. AJNR 1998;19:19-27.

9. Ducan JS, Sagar HJ. Seizure characteristics, pathology, and outcome after temporal lobectomy. Neurology 1987;37:405-409.

10. Bronen RA, Anderson AW. Quantitative MR for epilepsy: a clinical and research tool? AJNR 1994;15:1157-1160.

11. Baxendale AS: The hippocampus: functional and structural correlation. Seizure 1995;4:105-117.

12. Kuzniecky RI, Burgard S. Qualitative MRI segmentation in mesial temporal sclerosis: clinical correlations. Epilepsia 1996;37:433-439.

13. Wieshmann UC, Free SL. Magnetic resonance imaging in epilepsy with a fast FLAIR sequence. J Neurol Neurosurg Psychiatry 1996;61:357-361.

14. Fillipi M, Rocca MA, Wiessman M, et al. A comparison of MR imaging with fast FLAIR, HASTE-FLAIR and EPI FLAIR sequences in patients with multiple sclerosis. AJNR 1998;20:1931-1938. 